\title{
RFID Technology in Real Time Attendance Register System
}

\author{
Akiti Kenneth Tetteh ${ }^{a}$, Yeboah Derrick ${ }^{b}$, Amenya Bright $^{a}$, Li Shuguang ${ }^{a}$ \\ ${ }^{a}$ School of Automation, Zheijang University of Science and Technology, Hangzhou, China \\ ${ }^{b}$ College Oversea Education, Chengdu University, Chengdu, China \\ kennethakiti@gmail.com
}

\begin{abstract}
RFID stands for Radio Frequency Identification which provides the ability to identify, locate and sense the conditions of animate and inanimate entities. Radio frequency identification or RFID is a generic term for technologies that are used for auto identification of people or object using radio waves. In this technology a unique serial number is stored to identify a person or an object and other related information, on a microchip of silicon that is attached to an antenna which in turn is attached to a RFID reader. The antenna enables the chip to transmit the identification information to a reader then the reader converts the radio waves reflected back from the RFID tag into digital information that can then passed on to computers running RFID application middleware, that can make use of it. The system stores the absent and present student's attendance details in electronic format so that management of attendance becomes easy. Radio-Frequency Identification (RFID) is a technology that uses radio waves to transfer data from an electronic tag, called RFID tag or label, attached to an object, through a reader for the purpose of identifying and tracking the object. RFID technology which is a matured technology that has been widely deployed by various organizations as part of their automation systems. In this study, an RFID based system has been built in order to produce a time-attendance management system. This system consists of two main parts which include: the hardware and the software. The hardware consists of the motor unit and the RFID reader. The RFID reader, which is a low-frequency reader $(125 \mathrm{kHz})$, is connected to the host computer via a serial to USB converter cable. The Time-Attendance System GUI was developed using visual basic.Net. The Time-Attendance Management System provides the functionalities of the overall system such as displaying live ID tags transactions, registering ID, deleting ID, recording attendance and other minor functions. This interface was installed in the host computer.
\end{abstract}

Keywords: Systems, RFID, Technology, Attendance, Register,

DOI: $10.7176 / \mathrm{CEIS} / 12-2-06$

Publication date: September $30^{\text {th }} 2021$

\subsection{INTRODUCTION}

According to Co-build learner's dictionary Attendance is the fact that someone is present at an event or go regularly to an institution, or the attendance at an event is the number of people who are present at it. Furthermore, if someone is in attendance of a place or event, they are present. Empirical evidences have shown that there is a significant correlation between students' attendances and their academic performances. There was also a claim stated that the students who have poor attendance records will generally link to poor retention. Attendances of every student are being maintained by every school, college and university. Faculty has to maintain proper record for the attendance. The manual attendance record system is not efficient and requires more time to arrange record and to calculate the average attendance of each student. Hence there is a requirement of a system that will solve the problem of student record arrangement and student average attendance calculation.

\subsection{Motivation}

The purpose of this project is to facilitate the building of an attendance record system, which uses RFID as a means of attendance verification. The project also uses RFID technology as a communication platform, from the module to a central computer where attendance records will be stored. The project would help build a quality attendance system with available records which cannot easily compromised because it allows little human interferences. It would also be portable enough to be carried around by faculty and because of low cost be available for mass utilization by academic institution.

\subsection{LITERATURE REVIEW}

2.1 Introduction

Radio Frequency Identification (RFID) is an upcoming technology which has recently attracted the interest of the research community because of the extraordinary benefits it offers over the other existing identification and data capturing technologies. This chapter is formatted to review the existing RFID literature and explore the 
issues in the present RFID systems since the technology is still in its acceptance phase. Since the growth of RFID technology from 1900's, apart from its stated positive aspects, the technology also bears some concerns or issues. The intended purpose of this chapter is to examine the literature related to Radio Frequency Identification further extend academic research, and providing an insight into some of the outstanding and crucial issues hindering the growth of the RFID technology. There is a strong need to address these issues in order to provide a greater visibility and an increased product velocity of the RFID technology.

\subsection{The Evolution of RFID}

Radio Frequency Identification is a growing technology that has been around since early 1900's and was used in World War II. An early research paper had explored RFID work where the author of this paper stated that "Evidently, considerable research and development work has to be done before the field of useful applications is explored". Then, the electromagnetic theory related to RFID was studied in 1960's. Apart from that, inventions like Robert Richardson's "Remotely activated radio frequency powered devices" took place in that era. By this time, the wheels of RFID development had started turning. 1960's was the start of the adoption of RFID in commercial activities. A noticeable development work in this area had taken place in 1970's where vehicle tracking, factory automation etc. were the prime intentions. By 1980's, RFID technology had taken shape in terms of the full implementation of the technology. The deployment of applications using this technology was noticed in 1990's [86]. The pace of developments in RFID is as well apparent in the $21^{\text {st }}$ century where even the modest of item like cloth is bearing a small sticky patch of RFID and human implantation of RFID tag and that too of rice sized grain is the reality of the day [141].

\subsection{Technology Development}

At the first glance, it appears that the RFID technology is keeping its wheels turning without any difficulty since it might be a simple technology, but in reality it is not true. It is a technology that spans across diverse areas like CMOS design, data management, encryption, radio propagation, sensor design and integration, network engineering to mention a few. RFID technology makes use of components like tags and readers (normally connected to a host computer or network). The RFID tags are built using silicon chips and antennas. Based on being categorized as active or passive, RFID tags can be powered by a battery or illuminated by the radio waves emitted by a specialized reader respectively. The information gathered by an RFID interrogator or reader from an RFID tag is stored using a database system. A capacitor located in the circuit of the passive tag is responsible for storing power from the RFID reader. This collected energy is used to transmit the RFID tag information to the RFID reader. Selection amongst active and passive tags for use is a crucial component of the architectural design strategy. Architectural design of the RFID network is quite essential when it comes to development of an RFID system. Irrespective of the types of components used in an RFID system, the RFID system performance is evaluated based on how well it tracks objects. As per Solanas and Dominngo-Ferrer, it is crucial to craft a network that has the ability to scale in size. In the literature, a paper entitled "Developing a low cost RFID (Radio Frequency Identification) Middleware for Small Business Applications", it was concluded that "With RFID clearly emerging as the new supply chain standard, the opportunity is immense in this market". The paper also stated that "If any business is going to "win the game of tag", they must first understand how RFID can help their business and select RFID hardware and middleware that is not only applicable to their business but scalable for future growth.

\subsection{Spectrum Exploitation}

For RFID applications, the available spectrum of frequencies is quite limited. Moreover, it is taken care of or rather allocated by different national as well as regional regulatory authorities. Provided with this limited bandwidth and the variability of operating frequencies, the communication protocols between the RFID readers and the tags have to utilize the available spectrum efficiently. With time, as more and more RFID systems are implemented, the available bandwidth becomes over-utilized and collisions between readers and between tags increase, resulting in lower overall system performance. To address such concerns, research is on-going in the areas of tag anti-collision protocols. Simultaneous transmissions in RFID systems lead to collisions as the readers and tags operate on the shared wireless channel. Therefore, collision arbitration for RFID tags is a significant issue for fast identification. The collision problem occurs in signal transmission of the readers or the tags, which hardly leads to fast identification. Hence it becomes a key issue to develop an efficient anti-collision protocol so as to minimize collisions in the interrogation zone. The collision problems may be summarized into tag collision problems and the reader collision problems. In actual practical RFID systems, the former occur more often than the later. The tag collision problems are further sub-divided into active tag collisions and more complicated passive tag collisions. Since, the low-functional passive tags cannot detect collisions or figure out neighboring tags, it is of great significance to develop a tag anti-collision protocol improving the identification ability of RFID systems. 


\subsection{Data Storage and Management}

By providing the capability of automatic identification and data capture, the RFID technology can be used to significantly improve the efficiency of business processes. This section of the thesis focuses on the characteristics of RFID data and the challenges posed by RFID data. Through the automatic data collection provided by the RFID technology, RFID can achieve greater visibility and product velocity across supply chains, more efficient data management, easier product tracking and monitoring, reduced product counterfeiting. RFID poses many data related challenges and one of the biggest hills to climb is dealing with the flood of data RFID generates. For example, the in-store RFID implementation at Wal-Mart has the potential to generate as much data in three days as is contained in the entire U.S. Library of Congress. And it's not just a problem for the companies equal to the size of Wal-Mart, even the modest RFID deployments will generate gigabytes of rapidly changing data per day. Another most crucial factor resisting the high performance of RFID technology is the inaccuracy of the data streams generated by RFID readers. In real world RFID deployments, the observed rate of reading RFID tags is often in the range of $60-70 \%$. The observed data includes the correct data as well as the dirty data. This dirty data appears in the form of unreliable readings, missed readings and data redundancy. Getting the dirty data is very common in RFID applications and often happens in situations of low-cost, low power hardware and wireless communications. Hence, there is a demanding need to address ambient factors affecting the accuracy of RFID data leading to improved accuracy to determine the presence or absence of an RFID tag.

\subsection{Iris Recognition}

The human eye is sensitive to visible light. It will improve the lighting in the eye causes the pupil to contract to be great. Visible light causes specula reflections in the iris ring. In contrast, the human retina is less sensitive to near infrared radiation (NIR) in the wavelength range from $800 \mathrm{~nm}$ to $1400 \mathrm{~nm}$, but still detailed iris NIR imaging with illumination. In order for the measurement of dynamic movement to the human pupil, iris makes light reflex resulting from affecting the quality of digitalized images. This allows biological phenomena occur and develop two pupils illuminate simultaneously when one eye with visible light. This is an innovative method to propose the extraction of information on how the human eye responds to light. Decision several study show that it is this feature is the characteristics of distinguish. Although the uses of the Euclidean distance measurement, an average recognition accuracy of $99.1 \%$ is obtained. The method used has the potential to be a "fraud-proof" because the DFS can only be taken from the only living irises.

\subsection{Fingerprint Recognition System - Past To Present}

Fingerprint imaging technology has been in existence for centuries. Archaeologists have uncovered evidence suggesting that interest in fingerprints dates to prehistory. In Nova Scotia petroglyphs (from the time of pre-historic Native Americans) showing a hand with exaggerated ridge patterns has been discovered. In ancient Babylon and China, fingerprints were impressed on clay tablets and seals. The use of fingerprints as a unique human identifier dates back to second century B.C. China, where the identity of the sender of an important document could be verified by his fingerprint impression in the wax seal (Ruggles, 1996). In fourteenth-century Persia fingerprints were impressed on various official papers. At that time, a governmental official observed that no two fingerprints were exactly alike. Using the newly invented microscope, Professor Marcello Malpighi at the University of Bologna noted ridges on the surface of fingers in 1686. He described them as loops and spirals but did not note their value as a means of personal identification. Later, in 1823 at the University of Breslau, Professor John Evangelist Purkinje published his thesis proposing a system of classification based on 9 different fingerprint patterns. This was the first step towards the modern study of fingerprints. The first modern use of fingerprints occurred in 1856 when Sir William Herschel, the Chief Magistrate of the Hooghly district in Jungipoor, India, had a local businessman, Rajyadhar Konai, impress his handprint on the back of a contract. Later, the right index and middle fingers were printed next to the signature on all contracts made with the locals. The purpose was to frighten the signer of repudiating the contract because the locals believed that personal contact with the document made it more binding. As his fingerprint collection grew, Sir Herschel began to realize that fingerprints could prove or disprove identity. Despite his lack of scientific knowledge in fingerprinting he was convinced that fingerprints are unique and permanent throughout life. The French anthropologist, Alphonse Bertillon, devised the first widely accepted scientific method of biometric identification in 1870 .

The Bertillon system, Bertillon age, or anthropometry was not based on fingerprinting but relied on a systematic combination of physical measurements. These, among others, included measurements of the skull width, foot length, and the length of the left middle finger combined with hair color, eye color, as well as face and profile pictures. By grouping the data any single person could be placed into one of 243 distinct categories. For the next thirty years, Bertillon age was the primary method of biometric identification. Dr. Henry Faulds, British Surgeon-Superintendent of the Tsukiji Hospital in Tokyo, took up the study of fingerprints in the 1870's 
after noticing finger imprints on prehistoric pottery. In 1880, in the October 28 issue of the British scientific periodical Nature, Dr. Faulds was the first to publish a scientific account of the use of fingerprint as a means of identification. In addition to recognizing the importance of fingerprints, for identification he devised a method of classification as well. Dr. Faulds is credited for the first fingerprint identification-based on a fingerprint left on an alcohol bottle. The method of classification proposed by Dr. Faulds is called Henry Classification system and is based on patterns such as loops and whorls, which is still used today to organize fingerprint card files.

Continuing the work of Dr. Faulds, Sir William Herschel and Sir Francis Galton established the individuality and permanence of fingerprints. This book, "Fingerprints" from 1892, contains the first fingerprint classification system containing three basic pattern types: loop, arch, and whorl. The system was based on the distribution of the pattern types on the ten fingers. The system worked, but was yet to be improved with a classification that was easier to administer. Sir Galton identified the characteristics used for personal identification, the unique ridge characteristics known as minutiae, which are often referred to as "Galton's details". In 1892, Juan Vecetich, an Argentine Police official, made the first criminal fingerprint identification. He was able to identify a woman, who had murdered her two sons and cut her own throat in an attempt to avoid blame. Her bloody print was left on a doorpost, proving her identity as the murderer.

\subsubsection{Shift from Bertillion Age to Fingerprinting}

During the 1890's, Sir Edward Richard Henry, a British official in Bengal believed that a fingerprinting system was the solution to his problem of verifying the identity of criminals. He studied the works of Sir Galton and Sir Henry and proved that they could be used to produce 1,024 primary classifications, which was instituted in Bengal in 1897. The system is described in his book, "Classification and Uses of Finger Prints". In June 1897, Bertillon age was replaced and the Henry Classification System became the official method of identifying criminals in British India. In 1901, Sir Henry, established the first fingerprint files in London. Subsequently, within the next 25 years, the Henry Classification System was adopted as the universally accepted method of personal identification by law enforcement agencies throughout the world. It is still in use, though several variants of the Henry Classification System exist.

In 1903, the Henry classification system was used to differentiate two prisoners who were identical twins. The Bertillon system was not able to make out the difference between identical twins and thus Henry classification system was further strengthened. Juan Vucetich also worked on a classification system based on the findings of Sir Galton and years of experience in fingerprint forensics. His system was published in his book, "Dactiloscopía Comparada" (Comparative Fingerprinting) in 1904. His system, the Vucetich System, is still used in most Spanish-speaking countries. During the first 25 years of the 1900s, more and more agencies in the U.S. started to send copies of their fingerprint cards to the National Bureau of Criminal Identification. These files formed the nucleus of the FBI fingerprint files when the Identification Division of the FBI was established in 1924. By 1946, the FBI had processed more than 100 million fingerprint cards in manually maintained files. By 1971, this number had increased to 200 million cards. The first country to adopt a national computerized form of fingerprint imaging was Australia in 1986, which implemented fingerprint imaging technology into its law enforcement system. With the introduction of AFIS technology (Automated Fingerprint Identification System), the files were split into computerized criminal files and manually maintained civil files. Many files were found to be duplicated and the records actually represented somewhere between 25 and 30 million criminals and an unknown number of individuals in the civil files.

\subsection{METHODOLOGY}

\subsection{Proposed System}

The RFID attendance system is an automatic embedded system used in taking attendance of registered persons in a particular organization. The RFID attendance system offers an organization, the efficiency and convenience associated with RFID technology at a low cost. This method is fast as well as simple. Each Employee Uses an RFID Card and the Reader records the data when the employee enters or exits. RFID devices and software must be supported by a sophisticated software architecture that enables the collection and distribution of location based information in near real time. A complete picture of the RFID attendance system combines the RFID Tags and readers with access to global standardized database, ensuring real time access to up-to-date information on the card. The card contains a unique identification number called an electronic product code (EPC). Nowadays, there are lots of companies around the world and some of them consist of workers up to 10 thousand or more. To handle a large number of workers may be a problem specially to get the attendance of the workers. The manual process means that whenever a worker comes to work, he goes to sign at the time officer's table. This manual process has some flaws because in a case where a worker bribes the time officer or is familiar with him, the time officer may tamper with the attendance records. This would be a big problem in the company and might affect the productivity and management of the company. The suitable solution for this problem is by designing a system that will record attendance automatically. In this project, RFID system is used 
to record the numbers of employees' attendance automatically. The ID cards of the employees is embedded with RFID tag which is read by a reader. This RFID system is interfaced to a database through a computer.

\subsection{Structure of the Proposed System.}

A careful observation of the trend of usage of RFID tags leads one to consider the possibility of its utilization for monitoring the attendance of students in educational institutions, with the aid of program driven computers. While every student given a specific RFID tag attends the lecture through entrance door, a serial number (related to each student's matriculation number) of tag is associated with the student database entry. So every time a student uses his/her card, the entries will be entered into the database with the time stamp. The use of webcam might be optionally necessary. The tag is activated when it passes through a radio frequency (RF) field $(125 \mathrm{kHz}$ in this case), which is generated by the antenna embedded within the reader box. The program checks whether the tag is valid or not. If the tag is valid, it will continue to the database program and registers the student's attendance for the course. If the tag is invalid, the program gives a notification that the tag has not been registered to any student and requires the user to either supply a valid tag. Due to the reason of cost and flexibility of implementation, this RFID attendance design application uses a passive tag and thus for every class, students would have to bring their tags close to the reader (about $10 \mathrm{~cm}$ from the reader). On doing this, the reader reads the tag and the application program records the student's arrival time and when leaving the class, students will also have to bring their tags close to the reader again.

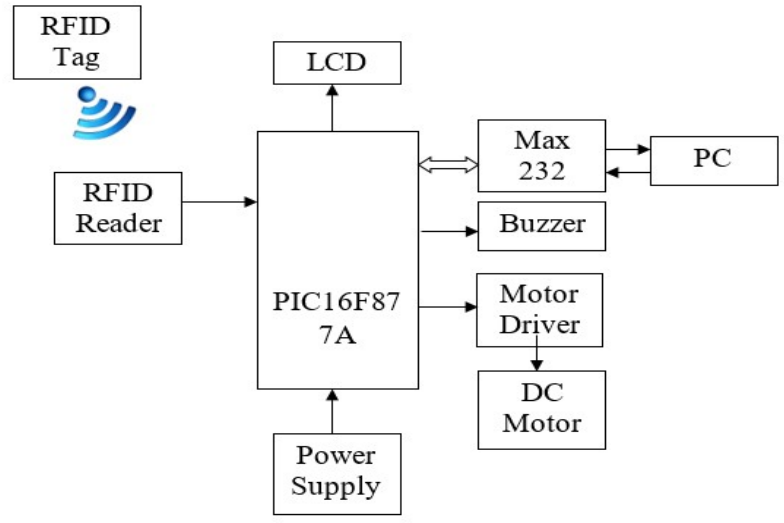

Figure 1: Block Diagram of RFID

\subsubsection{Description}

The project block diagram consists of four main blocks; Peripheral Interface Microcontroller, IR Sensor, Motor Drive, RFID. PIC microcontroller is the brain of the system. The input is given to the Controller from the IR sensor. The input is amplified using instrumentation amplifiers and fed to A/D converter. The output of the amplifier is fed to the series voltage regulator. The amplified output is given by the analog switch of the regulator. In our attendance register system, we use a microcontroller to manage the card reading operation and identifying a particular student. Before issuing the ID card the unique number for that particular card will be stored in the microcontroller and so that whenever you show to the RFID reader it will displays the presence of that particular student. The data will be monitored and stored in a personal computer. Power supply circuit used here provides $5 \mathrm{v}$ power for both microcontroller and the RFID reader, there will be a buzzer and an LED to indicate the RF is read. The RFID reader sends signals to the RS-232 circuit which interfaces with the serial port. The microcontroller is programmed to receive signals from the RS-232 circuit and sends signals to control the motor circuit which controls the direction of movement of the door. 


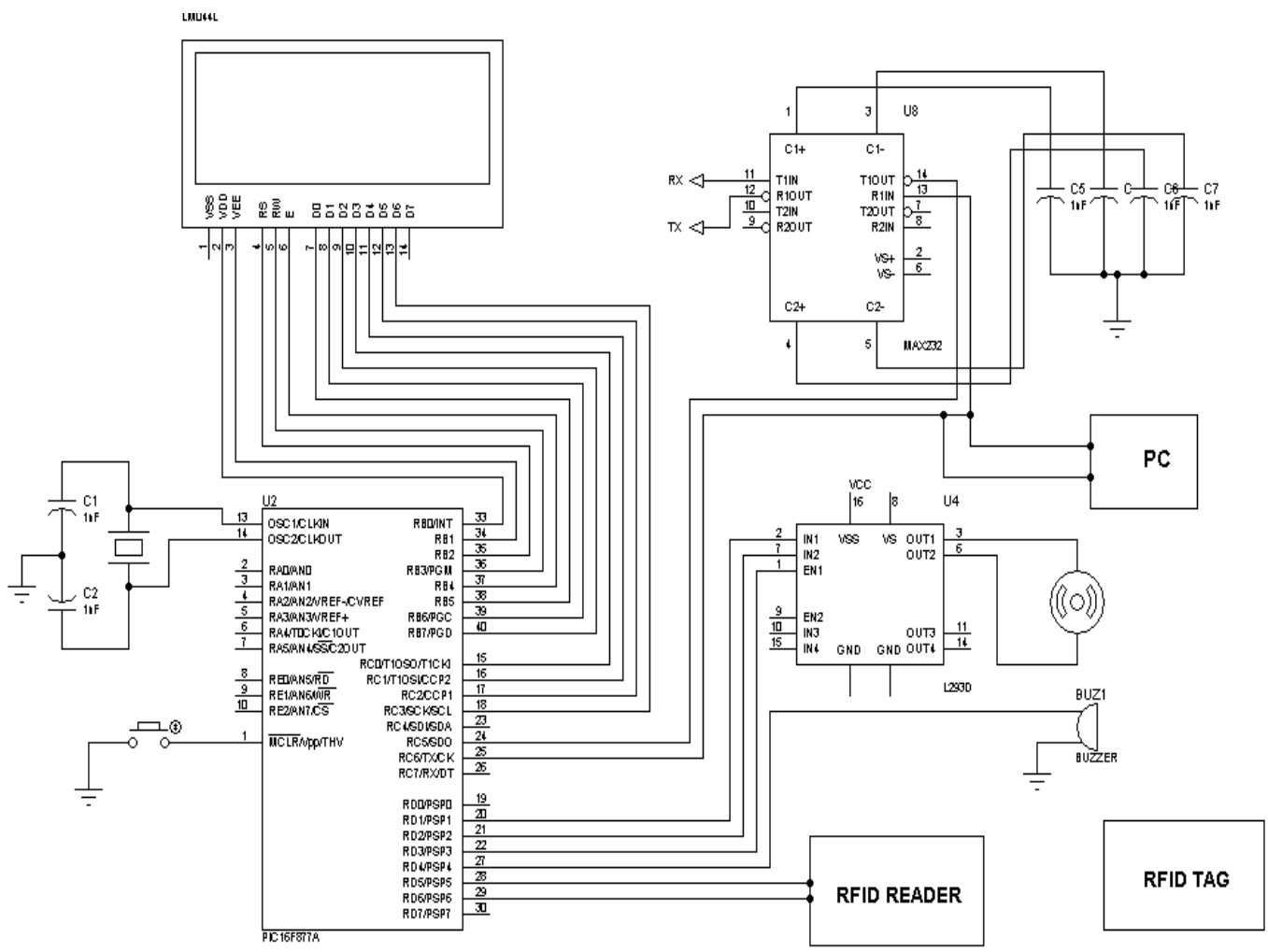

Figure 2: Circuit Diagram

\subsubsection{Circuit Diagram}

The motor interfaced with a designed database and graphical user interface(GUI) that will be responsible for taking and storing the data received from the reader. And the software development unit consisting the Microsoft visual studio, Microsoft .Net framework and Microsoft access. This is done by comparing the information received from the RFID reader to the information stored in the database. The microcontroller is programmed to receive signals from the RS-232 circuit and sends signals to control the motor circuit which controls the direction of movement of the door.

\subsection{PIC Microcontroller}

\subsubsection{PIC16F877A ARCHITECTURE}

PIC 16F877A is a 40-pin 8-Bit CMOS FLASH Microcontroller from Microchip. The core architecture is high-performance RISC CPU with only 35 single word1 instructions. Since it follows the RISC architecture, all single cycle instructions take only one instruction cycle except for program branches which take two cycles. 16F877 comes with 3 operating speeds with 4, 8, or $20 \mathrm{MHz}$ clock input. Since each instruction cycle takes four operating clock cycles, each instruction takes $0.2 \mu$ s when $20 \mathrm{MHz}$ oscillator is used. It has two types of internal memories: program memory and data memory. Program memory is provided by $8 \mathrm{~K}$ words (or $8 \mathrm{~K}^{*} 14$ bits) of FLASH Memory, and data memory has two sources. One type of data memory is a 368-byte RAM (random access memory) and the other is 256-byte EEPROM (Electrically erasable programmable ROM). The core feature includes interrupt capability up to 14 sources, power saving SLEEP mode, and (ICSP) capability. The sink/source current, which single 5V In-Circuit Serial Programming indicates a driving power from I/O port, is high with $25 \mathrm{~mA}$. Power consumption is less than $2 \mathrm{~mA}$ in $5 \mathrm{~V}$ operating condition. 


\subsubsection{The Peripheral Features Include:}

- time blocks: Timer0 for 8-bit timer/counter; Timer1 for 16-bit timer/counter; and Timer2: 8-bit timer/counter with 8-bit period register, prescaler and postscaler.

- $\quad$ Two Capture, Compare, PWM modules for capturing, comparing 16-bit, and PWM generation with 10bit resolution.

- $\quad$ 10-bit multi-channel $(\max 8)$ Analog-to-Digital converter module. (Master Mode) and I2

- Synchronous Serial Port (SSP) with SPI (Master/Slave) C2

- Universal Synchronous Asynchronous Receiver Transmitter (USART/SCI) with 9-bit address detection

- $\quad$ Parallel Slave Port (PSP) 8-bits wide, with external RD, WR and CS controls (g) I/O ports.

The key feature of $16 \mathrm{~F} 877 \mathrm{~A}$ is summarized below:

Table.5.1 Key feature of $16 F 877 A$

\begin{tabular}{|l|l|}
\hline FLASH Program Memory (14-bit word) & $8 \mathrm{~K}$ Words \\
\hline Data Memory (RAM) & 368 Bytes \\
\hline Data Memory (EEPROM) & 256 Bytes \\
\hline Interrupts & 14 \\
\hline I/O Ports & Ports A, B, C, D, E \\
\hline Timers & 3 \\
\hline Capture/Compare/PWM Modules & 2 \\
\hline Serial Communications & MSSP, USART \\
\hline Parallel Communications & PSP \\
\hline 10-bit Analog-to-Digital Module & 8 channels \\
\hline Instruction Set & 35 \\
\hline
\end{tabular}

\subsection{Overview of the PIC Peripheral}

The PIC16F877 is more than just a CPU with some RAM and ROM. There are also several useful peripheral hardware modules built-in to make it a complete computer control system. Many products can be made that use the PIC as a complete single-chip solution to its system design.

\section{1) Input/output Ports}

There are five port-sets as Ports (A, B, C, D, E) with bits and pins that may be set in software as inputs or outputs. These pins are also shared with other peripheral functions and to use these other functions requires setting up the input/output ports for compatibility. Thirty-three (33) input/output port pins are available.

\section{2) Interrupts}

As briefly noted in Chapter 6, an interrupt is a way for peripherals and other hardware to capture the attention of the CPU and have it call a subroutine to service the hardware with the user's software. The subroutine address is at a fixed location in program memory and is built-in to the CPU. There are a total of 14 possible interrupts and each of them can be enabled or disabled in software.

\section{3) Analog-to-Digital Converter \& the Analog Multiplexer}

The PIC contains a ten-bit ADC and has as many as eight available analog input channels. These analog inputs are shared with the port pins of Port A and Port E. It is also possible to select among these pins a place to attach an external voltage reference in the case where the user does not want to use the internal voltage reference of five volts. This feature is also software selectable.

\section{4) The Watch-Dog Timer}

The PIC contains its own independent watch-dog timer which can be disabled not by software but by a setting in the downloading process. The watch-dog timer has the power to reset the PIC when it overflows. The user's job in the software is to provide the watch-dog timer with regular commands to reset the watch-dog timer so that it will not reset the PIC. The purpose of the watch-dog timer is to make sure the software does not get trapped in infinity loops and thus the software is made much more reliable by using the watch-dog timer. In addition to the watch-dog timer is a pre-scaler which can extend the watch-dog's time-out period. 
5) Timer0, Timer1, and Timer 2

These three timers can count clock pulses or count external pulses on the PIC's port pins. They are programmable and have pre-scalers and sometimes post scalers to modify their counts and counting processes. They often serve as time-bases for other peripherals or for providing regular interrupts to the user's software.

6) Capture Mode (Two of them)

The capture mode modules are hardware-controlled ways to measure pulse-widths with reference to the system clock.

\section{7) Compare Mode}

The compare mode modules compare Timer1's count to a fixed, but user programmable, register value. When that value is reached, the hardware can send out a pulse, trigger an interrupt, or do some kind of "special event" within the PIC such as resetting and reloading Timer1 and/or starting the ADC. This is also useful for setting up a time-base for the software.

\section{8) Pulse-Width Modulation}

The PWM modules use Timer2 to generate signals on a PIC output pin and serve as DACs. Both PWM modules have a maximum of ten bits of resolution.

9) Parallel Slave Port

Port D can be used as a bi-directional, microprocessor-type, data-bus port. The PIC is controlled as a slave to three external control signals which are manipulated by the microprocessor or other device.

\section{0) EEPROM Data Memory}

The PIC has 256 bytes of non-volatile EEPROM and can be programmed in the PIC software independent of a device programmer.

\section{1) FLASH Program Memory}

In a similar way to the EEPROM Data Memory, the FLASH Program Memory can also be programmed in software independent of a device programmer. New program features and updates can be downloaded to the PIC's software while the unit is in the field.

\section{2) Code and Data Protection}

The PIC can be configured to lock-out attempts to read or write its FLASH and EEPROM Data Memories. This can be set only during the downloading process.

\section{3) Resets and Sleep Mode}

The PIC has several modes of resets and status conditions to identify which of them has occurred. Also, there is a power-saving mode called "sleep" which can be initiated in software to power-down the CPU. This is useful in battery powered applications.

\section{Input/output Ports}

There are five input/output ports as Port (A, B, C, D, E). Most of these act alike but some are different and use different features and options. Port a There are six Port A pins and each can be set as an input or an output. All of the Port A pins except RA4 have a shared use with the analog multiplexer (MUX). The RA4 pin can be a Schmitt Trigger input (hysteresis) or an open-drain output. The first step in configuring Port A is to select which pins are to be set as digital input/output and which are to be analog inputs. This is done with the lower four bits of the ADCON1 register file (RAM). If we want all of Port A to be digital, the setting is 0x06. Of the pins which are digital input/output the selection of "input" vs. "output" is made with the TRISA register file. If a Port A pin is to be an "input", the TRISA bit corresponding to the Port A pin/bit must be set to one (=1). If it is to be an "output", the TRISA bit must be set to zero $(=0)$.

\section{Port B, Port C, Port D}

None of Port B, Port C, or Port D is shared with the analog multiplexer and none need be configured with the ADCON1 register file. There are TRISB, TRISC, and TRISD register files which control the "input" vs. "output" selections of each of these port pins. These work in the same way as Port A. The Port B pins also have a user selectable "weak pull-up" option that can be enabled by clearing the "/RBPU" bit of the "OPTION_REG" register file. (Do "BCF OPTION_REG, RBPU".) This feature is automatically disabled when a Port B pin is configured as an output. 
The Port E pins also share their pins with the analog multiplexer as in Port A and its use is identical with Port A. (Use the ADCON1 and TRISE register files.)

\section{Interrupts}

The PIC16F877 has a total of 14 sources of interrupts. Each of these has an "Interrupt Enable Bit" which must be set $(=1)$ to enable or use the interrupt and an "Interrupt Flag", which is set $(=1)$ automatically when the interrupt is activated. There is also a "Global Interrupt Enable Bit" and a "Peripheral Interrupt Enable Bit". The "Global" must be set $(=1)$ before any of the other interrupts will be enabled. The "Peripheral" must be set $(=1)$ before any peripheral interrupt will be enabled. When an interrupt occurs the CPU treats it like a subroutine "CALL" and the program-counter is set for the program address, 0x0004. The user must place the interrupt-handling subroutine at this address (this subroutine is called, the "Interrupt Service Routine" or "ISR"). A special instruction is used to return from an ISR: It is the "RETFIE" instruction. Before returning from an interrupt, the user must reset the "Interrupt Flag" which was set $(=1)$ when the interrupt occurred. If this is not done, the interrupt hardware will automatically and immediately cause another interrupt to occur when the CPU executes the "RETFIE" instruction! It is also possible to inadvertently cause an interrupt to occur when the user sets $(=1)$ an "Interrupt Enable Bit". If the corresponding "Interrupt Flag" is set $(=1)$ when the user sets the "Interrupt Enable Bit", an inadvertent or accidental interrupt will occur!

\section{$A D C$ and Analog $M U X$}

The PIC's ADC can convert an analog voltage to a ten-bit number. The analog multiplexer (MUX) will allow up to 8 analog input channels to be converted by the ADC. The voltage range limits may be taken internally as " +5 Volts and Ground", or the user can supply an external voltage reference on the analog input channel pins. The ADC module uses two control registers in the register file map to set-up the ADC and the analog MUX. These are "ADCON0" and "ADCON1". The ADC may be used with or without interrupts. The first step in using the ADC is to set-up the analog MUX inputs which are shared with Port A and Port E. This was discussed under using Port A by selecting a code and putting that four-bit code into the lower four bits of "ADCON1". For the selected analog MUX inputs, the TRISA and TRISE registers must be set-up so that those Port A and Port E pins are configured as "inputs". If they are configured as "outputs", a voltage of either five volts or ground will jam the ADC. The next step is to select the ADC conversion clock rate using the "ADCS1" and "ADCS0" bits of the "ADCON0" register.

\section{Watch-Dog Timer}

The watch-dog timer built-in to the PIC runs with its own RC oscillator and has a typical minimum time-out period of 7 milliseconds which in general is too short an amount of time. There is a programmable prescaler available that can multiply this period by 128 to give a total time-out period of 850 milliseconds, which is very good for most applications. The software must reset the watch-dog timer before it overflows and resets the PIC. This is done with the "CLRWDT" instruction. If the watch-dog timer does reset the PIC, there is a "STATUS" bit, "/TO", which indicates that this has occurred. If "/TO" = Zero $(=0)$, the watch-dog timer has reset the PIC. The watch-dog timer can be enabled or disabled using the "CONFIGURATION" word or in the down-loading process. The watch-dog timer should NEVER be disabled! Also, NEVER use an Interrupt Service Routine to reset the watch-dog timer!

Timer0

Timer0 is an 8-bit counter/timer which can be driven from the Fosc/4 clock, a pre-scaled Fosc/4 clock, or an external pin source called, "T0CKI", which is shared with Port A, RA4. Timer0 is readable and write-able and its output can generate an interrupt, "T0IF". If Timer0 is used as a counter (with "T0CKI" input), the user can select if it should count on the rising edge or the falling edge. The programmable pre-scaler may be used on either the timer mode or the counter mode. This pre-scaler is the same one that is used by the watch-dog timer and it cannot be used by both Timer0 and the watch-dog timer at the same time. Since the "T0CKI" input is shared with the Port A, RA4 pin, the user must set-up Port A, RA4 as an "input" with TRISA, if the counter mode is to be used.

\section{Sleep Modes \& Reset Modes}

The PIC has a power-saving mode where the CPU can be deactivated only to be re-activated later but without losing RAM data or register data. This is called the "Sleep" mode and it is initiated by executing the "SLEEP" instruction in the user's software. Processor "resets" and interrupts can take the PIC out of "Sleep" 
mode. These will be discussed shortly. There are several kinds of processor "resets" in the PIC. A list of these is as follows:

1) Power-On Reset (POR)

2) /MCLR --- Normal Operation

3) /MCLR --- From Sleep

4) Watch-Dog Timer (WDT) --- Normal Operation

5) Watch-Dog Timer (WDT) --- From Sleep

6) Brown-Out Reset (BOR)

The "power-on reset" can be enabled to wait until the DC power has come up to a safe level before initializing the PIC. Likewise, the "brown-out reset" can be enabled to look for drops in the DC voltage powering the PIC and cause a reset to occur. The "power on" reset, the "brown-out" reset, and the watch-dog timer are all enabled or disabled from the CONFIGURATION word. The "/MCLR" reset pin (pin 1) is held high with a pull-up in normal operation. Each of these resets has "flag" or "status" bits that allow the user to detect which of these reset has occurred from software. These bits are located in the "PCON" and "STATUS" registers.

The PIC has three major serial communications modes:

1) USART

The USART is a "Universal Synchronous/Asynchronous Receiver/Transmitter". It performs RS-232 serial communications with the IBM-PC serial port when it is operated in its asynchronous mode. It can also work in a master or slave synchronous mode but only in a half-duplex form.

\section{2) Master Synchronous Serial Port, Serial Peripheral Interface}

The MSSP/SPI mode is a simple 8-bit serial input/output used for working with shift-registers and other simple serial interfaces. It is not used for RS-232. Like the USART, it also has a master or slave mode.

\section{3) Master Synchronous Serial Port, Inter-Integrated Circuit}

The MSSP/I2C mode is a more complex serial communications mode that is supported by many off-the-shelf integrated circuits. It is intended for more complex systems where there are several master mode devices and many slave mode devices. There is a complicated communications protocol that links each master to each slave. It is not used for RS-232.

\subsection{RF Reader}

Introduction: RFID READER AND CARD

The conventional method of tracking an object, monitoring maintenance of database involves recording the necessary details manually. This manual method has the following disadvantages:

$>$ Prone to error

$>$ Highly time consuming

$>$ Tedious

$>$ Unreliable

Manipulation of entries is possible

An advanced automatic identification system known as Radio Frequency Identification technology (RFID) is a remedial measure which would eliminate the problems of the conventional system. RFID consists of electrical device called RFID tags which stores the unique information about the person and transmits this information continuously. The transceivers at different locations pick up the necessary information from the tag.

\section{RFID Technology- An Overview}

RFID stands for radio frequency identification. It is an automatic identification technology to identify objects by using invisible radio waves. Instead of optically scanning bar codes on a label, RFID uses radio waves to capture data from tags. One of the key characteristics of RFID is that it does not require the tag to be seen to read the data stored in it. This means that the tag can be placed either inside or outside. To accomplish this, 2 components are essential: reader and a tag. 


\section{Basic Elements of RFID}

\section{Tag}

2. Readers

TAG:

A tag, also called as transponder, is made of a micro-chip with a coiled antenna. The tag can be programmed with unique information about the object and hence can be used to identify it. RFID tags can be encased in hardened plastic coatings making them extremely durable.

RFID tags can store large amounts of data. High end RFID tags can store up to 1 megabyte of data. Some RFID tags supports read/write operations, enabling real time information updates as the tagged item moves from one location to another.

\section{TYPES OF RFID TAGS}

\section{PASSIVE}

2. ACTIVE

\section{PASSIVE:}

Passive RFID tags have no internal power supply. The minute electrical current induced in the antenna by the incoming radio frequency signal provides just enough power for the CMOS integrated circuit in the tag to power up and transmit a response. Most passive tags signal by backscattering the carrier signal from the reader. This means that the antenna has to be designed to both collect power from the incoming signal and also to transmit the outbound backscatter signal. The response of a passive RFID tag is not necessarily just an ID number; the tag chip can contain non-volatile EEPROM for storing data.

\section{ACTIVE:}

Unlike passive RFID tags, active RFID tags have their own internal power source which is used to power any ICs that generate the outgoing signal. Active tags are typically much more reliable (e.g. fewer errors) than passive tags due to the ability for active tags to conduct a "session" with a reader. Active tags, due to their onboard power supply, also transmit at higher power levels than passive tags, allowing them to be more effective in "RF challenged" environments like water (including humans/cattle, which are mostly water), metal (shipping containers, vehicles), or at longer distances. Many active tags have practical ranges of hundreds of meters, and a battery life of up to 10 years. Some active RFID tags include sensors such as temperature logging which have been used in concrete maturity monitoring or to monitor the temperature of perishable goods. Other sensors that have been married with active RFID include humidity, shock/vibration, light, radiation, temperature and atmospherics like ethylene. Active tags typically have much longer range (approximately 300 feet) and larger memories than passive tags, as well as the ability to store additional information sent by the transceiver. The United States Department of Defense has successfully used active tags to reduce logistics costs and improve supply chain visibility for more than 15 years. At present, the smallest active tags are about the size of a coin and sell for a few dollars.

\section{READERS:}

The RFID reader is designed for fast and easy system integration without losing performance, functionality or security. The RFID reader consists of a real time processor, operating system, virtual portable memory, and transmitter/receiver unit in one small self-contained module that is easily installed in the ceiling or in any other convenient location. A reader, also called interrogator, comprises of a transmitter, receiver, control module and a transceiver. The transceiver acts as a communication link between the person and the controlling PC. A reader should have an attached antenna which is used to transmit and receive the RF signal. Each reader has associated software that allows the user to read and program the tags.

\section{THE RFID System:}

An RFID system may consist of several components: tags/transponders, tag readers, antenna, middleware/application software. The purpose of an RFID system is to enable data to be transmitted by a mobile device, called a tag, which is read by an RFID reader and processed according to the needs of a particular application. The data transmitted by the tag may provide identification or location information, or specifics about the product tagged, such as price, color, date of purchase, etc. The use of RFID in tracking and access applications first appeared in 1932, to identify aircraft as friendly or unfriendly ("identify friend or foe" (IFF)). RFID quickly gained attention because of its ability to track moving objects. As the technology is refined, more 
pervasive and possibly invasive uses for RFID tags are in the works. In a typical RFID system, individual objects are equipped with a small, inexpensive tag. The tag contains a transponder with a digital memory chip that is given a unique electronic product code. The interrogator, an antenna packaged with a transceiver and decoder, emits a signal activating the RFID tag so it can read and write data to it. When an RFID tag passes through the electromagnetic zone, it detects the reader's activation signal. The reader decodes the data encoded in the tag's integrated circuit (silicon chip) and the data is passed to the host computer. The application software on the host processes the data, and may perform various filtering operations to reduce the numerous often redundant reads of the same tag to a smaller and more useful data set.

\section{Operating Principles of RFID:}

There is a huge variety of different operating principles for RFID systems. The picture below provides a short survey of known operation principles. The most important principle - 'inductive coupling' is described below

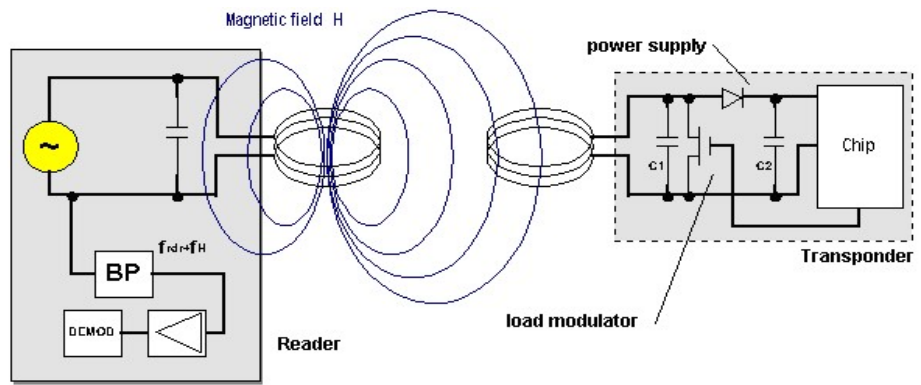

Figure 3: Operating Principles of RFID

As described above, inductively coupled systems are based upon a transformer-type coupling between the primary coil in the reader and the secondary coil in the transponder. This is true when the distance between the coils does not exceed, so that the transponder is located in the 0.16 near field of the transmitter antenna. If a resonant transponder (i.e. the self-resonant frequency of the transponder corresponds with the transmission frequency of the reader) is placed within the magnetic alternating field of the reader's antenna, then this draws energy from the magnetic field. This additional power consumption can be measured as voltage drop at the internal resistance in the reader antennae through the supply current to the reader's antenna. The switching on and off of a load resistance at the transponder's antenna therefore effects voltage changes at the reader's antenna and thus has the effect of an amplitude modulation of the antenna voltage by the remote transponder. If the switching on and off of the load resistor is controlled by data, then this data can be transferred from the transponder to the reader. This type of data transfer is called load modulation. To reclaim the data in the reader, the voltage measured at the reader's antenna is rectified. This represents the demodulation of an amplitude modulated signal.

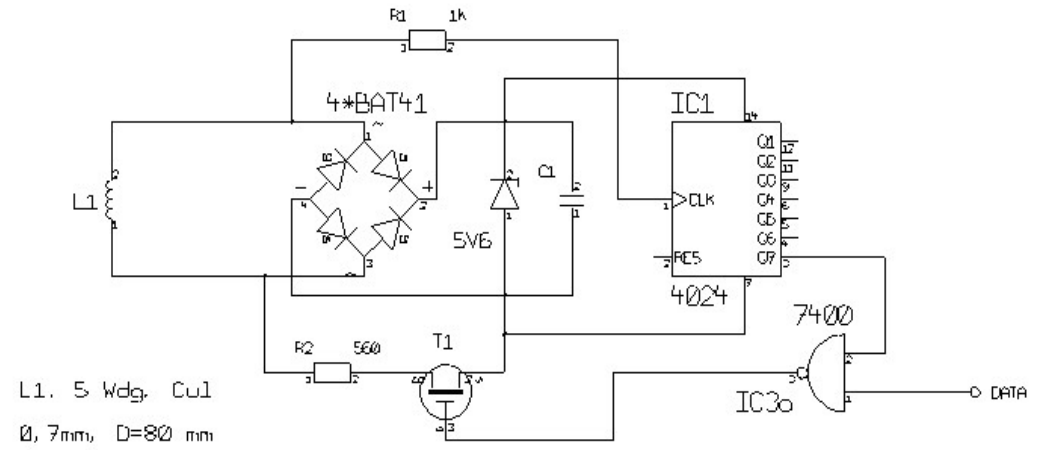

Figure 4: Sample Circuit of the Power Supply and Load Modulator in a Transponder

If the additional load resistor in the transponder is switched on and off at a very high elementary frequency $\mathrm{fH}$, then two spectral lines are created at $\mathrm{f} \square \mathrm{a}$ distance of $\mathrm{H}$ around the transmission frequency of the reader, and 
these can be easily detected (however fH must be less than fREADER). In the terminology of radio technology, the new elementary frequency is called a subcarrier. Data transfer is by the ASK, FSK or PSK modulation of the subcarrier in time with the data flow. This represents an amplitude modulation of the subcarrier.

\section{Advantages OF RFID:}

The advantages of RFID are:

$>$ Large amounts of data can be transmitted at very high speeds

$>$ Manual work is almost eliminated

$>$ Facilitates error free mechanism

$>$ Highly reliable

\subsubsection{Areas of Application for RFID:}

Potential applications for RFID may be identified in virtually every sector of industry, commence and services where data is to collect. The attributes of RFID are complimentary to other data capture technologies and thus able to satisfy particular application requirements that cannot be adequately accommodate by alternative technologies. Principal areas of applications for RFID that can be currently identified include :

$>$ Transportation and logistics

$>$ Manufacturing and processing

$>$ Security

A range of miscellaneous application may be distinguished, some of which are steadily growing in items of application numbers. They include:

$>$ Animal tagging

$>$ Waste management

$>$ Time and Attendance

$>$ Postal Tracking

$>$ Airline Baggage Reconciliation

$>$ Road Toll Management

As standards emerge, technology develops still further, and costs reduce considerable growth in terms of application numbers and new areas of applications may be expected.

Some of the More Prominent Specific Application Includes:

$>$ In Singapore, the public transport network of buses and trains employs passive RFID cards known as EZLink cards.

$>$ RFID tags are being embedded in passports issued by many countries. The first RFID passports ("epassports") were issued by Malaysia in 1998. In addition to information also contained on the visual data page of the passport, Malaysian e-passports record the travel history (time, date, and place) of entries and exits from the country.

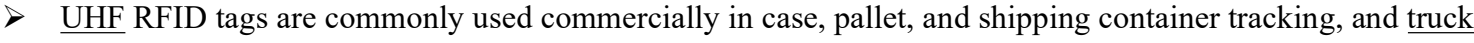
and trailer tracking in shipping yards.

$>$ Microwave RFID tags are used in long range access control for vehicles 
$>$ Implanted RFID tags are also used for animal identification.

A number of factors influence the suitability of RFID for given applications. The application needs must be carefully determined and examined with respect to the attributes that RFID and other data collection technologies can offer. Where RFID is identified as a contender further considerations have to be made in respect of application environment, from an electromagnetic standpoint, standards, and legislation concerning use of frequencies and power levels.

\subsection{LCD Display}

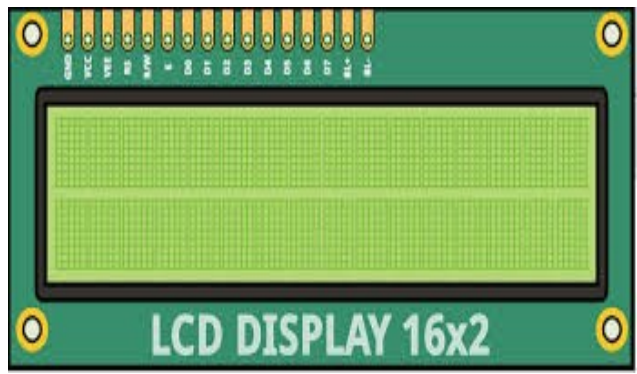

Figure 5: LCD Display

Alphanumeric displays are used in a wide range of applications, including palmtop computers, word processors, photocopiers, point of sale terminals, medical instruments, cellular phones, etc. The 16 x 2 intelligent alphanumeric dot matrix display is capable of displaying 224 different characters and symbols. A full list of the characters and symbols is printed on pages 7/8 (note these symbols can vary between brand of LCD used). This booklet provides all the technical specifications for connecting the unit, which requires a single power supply $(+5 \mathrm{~V})$. Available as an optional extra is the Serial LCD Firmware, which allows serial control of the display. This option provides much easier connection and use of the LCD module. The firmware enables microcontrollers (and microcontroller based systems such as the PICAXE) to visually output user instructions or readings onto an LCD module. All LCD commands are transmitted serially via a single microcontroller pin. The firmware can also be connected to the serial port of a computer.

3.9 DC Motor Driver (L293D)

L293D is a dual H-bridge motor driver integrated circuit (IC). Motor drivers act as current amplifiers since they take a low-current control signal and provide a higher-current signal. This higher current signal is used to drive the motors.L293D contains two inbuilt H-bridge driver circuits. In its common mode of operation, two DC motors can be driven simultaneously, both in forward and reverse direction. The motor operations of two motors can be controlled by input logic at pins $2 \& 7$ and $10 \& 15$. Input logic 00 or 11 will stop the corresponding motor. Logic 01 and 10 will rotate it in clockwise and anticlockwise directions, respectively. Enable pins 1 and 9 (corresponding to the two motors) must be high for motors to start operating. When an enable input is high, the associated driver gets enabled. As a result, the outputs become active and work in phase with their inputs. Similarly, when the enable input is low, that driver is disabled, and their outputs are off and in the highimpedance state.

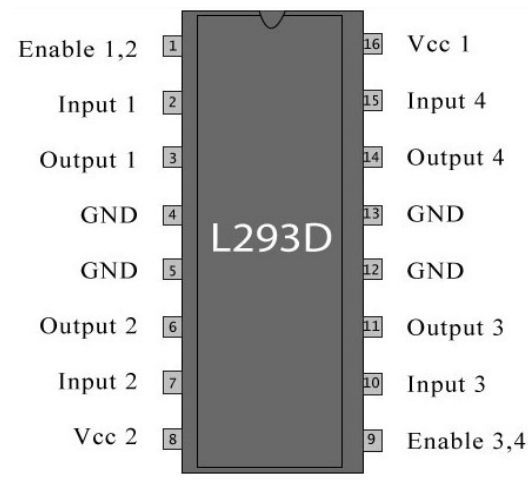

Figure 6: 3.9.1 Pin Diagram 


\section{Pin Description:}

Table: Pin Description

\begin{tabular}{|l|l|l|}
\hline Pin No & Function & Name \\
\hline 1 & Enable pin for Motor 1; active high & Enable 1,2 \\
\hline 2 & Input 1 for Motor 1 & Input 1 \\
\hline 3 & Output 1 for Motor 1 & Output 1 \\
\hline 4 & Ground (0V) & Ground \\
\hline 5 & Ground (0V) & Ground \\
\hline 6 & Output 2 for Motor 1 & Output 2 \\
\hline 7 & Input 2 for Motor 1 & Input 2 \\
\hline 8 & Supply voltage for Motors; 9-12V (up to 36V) & Vcc $2^{2}$ \\
\hline 9 & Enable pin for Motor 2; active high & Enable 3,4 \\
\hline 10 & Input 1 for Motor 1 & Input 3 \\
\hline 11 & Output 1 for Motor 1 & Output 3 \\
\hline 12 & Ground (0V) & Ground \\
\hline 13 & Ground (0V) & Ground \\
\hline 14 & Output 2 for Motor 1 & Output 4 \\
\hline 15 & Input2 for Motor 1 & Input 4 \\
\hline 16 & Supply voltage; 5V (up to 36V) & Vcc 1 \\
\hline
\end{tabular}

\subsection{MOTOR}

\subsubsection{Construction}

A permanent magnet is positioned around a loop of wire that is connected to a D.C. power source. The ends of the wire loop are connected to a set of contacts called the commutator, which rubs against a set of conductors called the brushes. The brushes make electrical contact with the commutator as it spins, and are connected to the positive and negative leads of the power source, allowing electricity to flow through the loop. The electricity flowing through the loop creates a magnetic field that interacts with the magnetic field of the permanent magnet to make the loop spin. DC motors consist of rotor-mounted windings (armature) and stationary windings (field poles). In all DC motors, except permanent magnet motors, current must be conducted to the armature windings by passing current through carbon brushes that slide over a set of copper surfaces called a commutator, which is mounted on the rotor. The commutator bars are soldered to armature coils. The brush/commutator combination makes a sliding switch that energizes particular portions of the armature, based on the position of the rotor. This process creates north and south magnetic poles on the rotor that are attracted to or repelled by north and south poles on the stator, which are formed by passing direct current through the field windings.

\subsubsection{Principles of Operation}

In any electric motor, operation is based on simple electromagnetism. A current-carrying conductor generates a magnetic field; when this is then placed in an external magnetic field, it will experience a force proportional to the current in the conductor, and to the strength of the external magnetic field. As you are well aware of from playing with magnets as a kid, opposite (North and South) polarities attract, while like polarities (North and North, South and South) repel. The internal configuration of a DC motor is designed to harness the magnetic interaction between a current-carrying conductor and an external magnetic field to generate rotational motion. Let's start by looking at a simple 2-pole DC electric motor (here red represents a magnet or winding with a "North" polarization, while green represents a magnet or winding with a "South" polarization).

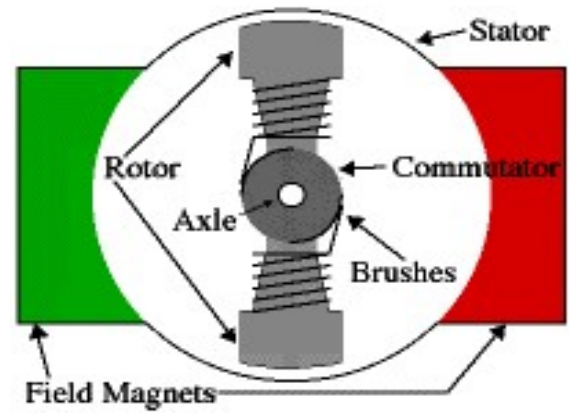

Figure 7: DC Motor 
Every DC motor has six basic parts -- axle, rotor (a.k.a., armature), stator, commutator, field magnet(s), and brushes. In most common DC motors (and all that Beamers will see), the external magnetic field is produced by high-strength permanent magnets. The stator is the stationary part of the motor -- this includes the motor casing, as well as two or more permanent magnet pole pieces. The rotor (together with the axle and attached commutator) rotates with respect to the stator. The rotor consists of windings (generally on a core), the windings being electrically connected to the commutator. The above diagram shows a common motor layout -- with the rotor inside the stator (field) magnets. After the building of the system, the aims and objectives were achieved to a suitable level. The circuit functioned as planned and designed. After much drawbacks, we were able to finally achieve the aims and objectives to a suitable level.

\subsection{RESULTS AND CONCLUSION:}

At the conclusion of a study made by the Iris and fingerprint system, RFID system selection to choose from. This is because the two systems studied have some disadvantages compared to the RFID system. Selection as a RFID attendance system is selected based on the problems and objectives that have been made. In conclusion, the objective to build an RFID based attendance system with a door unit was successfully achieved. In terms of performance and efficiency, this project has provided a convenient method of attendance marking compared to the traditional method of attendance system. By using databases, the data is more organized. This system is also a user friendly system as data manipulation and retrieval can be done via the interface, making it a universal attendance system. Thus, it can be implemented in either an academic institution or in organizations. However, some further improvements can be made on this RFID in order to increase its reliability and effectiveness. By incorporated an indicator or an LCD screen in the system to indicate when an unregistered card is scanned.

\section{Reference:}

[1] Daniel M. Dobkin and Steven M. Weigand (2010), "Environmental effects on RFID Tagantennas", Bulis press, California.

[2] SEEED RFID Reader Manual, www.seeedstudio.com.

[3] Julia Case and Anita C. Millspaugh (2005), "Programming in visual basic. NET" Mcgraw press, Sao Paulo.

[4] Financial Consortium Publishes RFID Standards for IT Assets. Retrieved on 2010.

[5] Amirjan Bin Nawabjan (2009), “Automated Attendance Management Software”, University of technology, Malaysia: degree thesis

[6] Stevan Preradovic and Nemai C. Karmakar (2006), "RFID reader: a review", Monash University, Australia

[7] Carlos. C. Murphy (2005), “Automated Attendance Systems”, Javier pressm, Argentina

[8] Mohd Firdaus Bin Mahyidin (2008), “students’ Attendance System Using RFID Technology”, Malaysia.

[9] Billard F. Cookes (2001), "Fundamentals on Barcode Technology", Rafot press ltd, Cyprus

[10] Gordon .N. With taker (2002), "introduction to Biometrics Technology", Dint and sons ltd, England.

[11] Markus Hansen and Sebastian Meissner (2000), "Identification and Tracking of Individuals and Social

Networks using the Electronic Product Code on RFID Tags", IFIP Summers 1td, Sweden

[12] Greene. C. Thomas (2004), "Feds approve human RFID implants",

http://www.theregister.co.uk/2004/10/14/human_rfid_implants/. Retrieved 